\title{
Editorial
}

Neuro[mmunoModulation

\section{Immunology of Psychiatric Disorders}

\author{
Antonio Lucio Teixeira ${ }^{a} \quad$ Norbert Müller ${ }^{b}$ \\ aLaboratório Interdisciplinar de Investigação Médica, Faculdade de Medicina, Instituto de Estudos Avançados \\ Transdisciplinares, Universidade Federal de Minas Gerais (UFMG), Belo Horizonte, Brazil; ${ }^{b}$ Department of Psychiatry \\ and Psychotherapy, Ludwig-Maximilian University of Munich, Munich, Germany
}

Recent reports on the Global Burden of Diseases, Injuries and Risk Factors Study 2010 [1, 2] demonstrated the significant burden associated with psychiatric disorders, with growing challenges for health systems. For instance, psychiatric disorders, including substance use disorders, were the leading cause of years lived with disability (YLD) worldwide, accounting for $22.9 \%$ of all YLD [2]. To change this picture the prevention and treatment of psychiatric disorders must be regarded as a social priority.

Complicating the scenario, the advancement of knowledge on the neurobiology of psychiatric disorders has not been translated into new or more effective pharmacological interventions. Facing growing costs and the exhaustion of amine-based strategies, part of the pharmaceutical industry has even withdrawn research and development in psychiatry. Furthermore, the promises of understanding the genetic basis of individual psychiatric disorders with genome-wide association studies did not materialize as shared genetic etiology for major psychiatric disorders was reported [3].

The immune system is traditionally regarded as a host defense system. In recent years, however, its homeostatic role has been emphasized [4]. The immune system works in association with other systems, particularly nervous and endocrine systems, to maintain body homeostasis. Corroborating this view, immune changes have been described in association with several noninfectious conditions, like physical exercise, weight gain/obesity, aging and psychiatric disorders.

The initial approach to the role of the immune system in behavior dates back to the study of 'sickness behavior', i.e. a set of depressive-like cognitive and behavioral

\section{KARGER}

E-Mail karger@karger.com

www.karger.com/nim changes that take place after an inflammatory condition. Subsequently, great interest was directed towards investigating a pathogenic role of infectious (e.g. toxoplasmosis) and noninfectious (e.g. autoimmunity) mediated inflammation in psychiatric disorders. Now, immune system-related molecules are being regarded either as putative biomarkers or as pharmacological targets.

This supplement of Neuroimmunomodulation presents the 'state of the art' of the immunology of psychiatric disorders. The manuscripts discuss the available data on the characterization of the immune/inflammatory dysfunction in the most relevant psychiatric disorders, associations with cognitive and behavioral symptoms, and potential biomarkers and targets for therapeutic development.

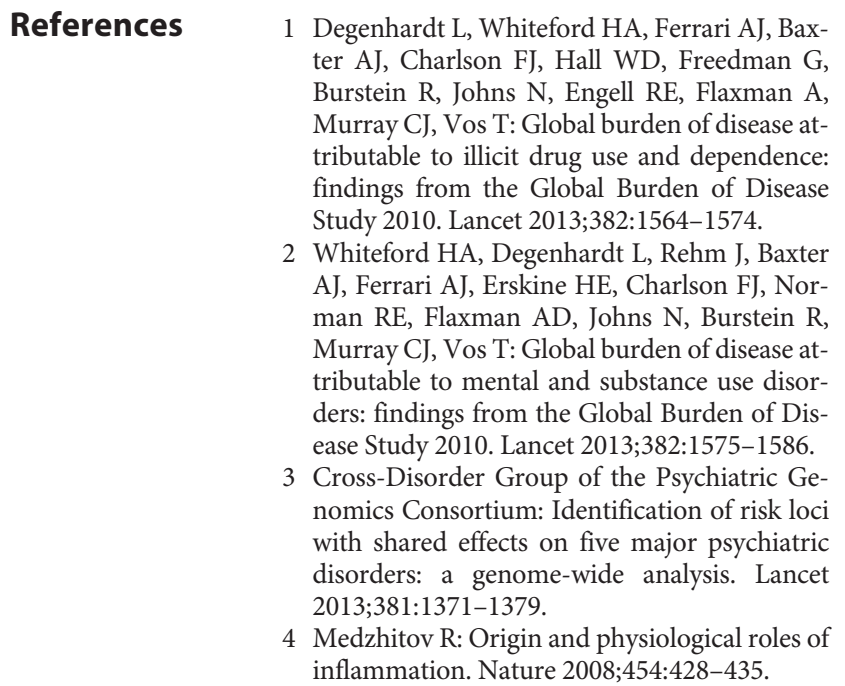

Antonio Lucio Teixeira

Laboratório Interdisciplinar de Investigação Médica, Faculdade de Medicina Av. Alfredo Balena, 190, Sala 281

Santa Efigênia, Belo Horizonte 30130-100 (Brazil)

E-Mail altexr@gmail.com 